\title{
Pretreatment imaging of peripheral vascular malformations
}

This article was published in the following Dove Press journal:

Journal of Vascular Diagnostics

3 October 2014

Number of times this article has been viewed

\author{
Joshua B Johnson \\ Petrice M Cogswell \\ Michael A McKusick \\ Larry A Binkovitz \\ Stephen J Riederer \\ Phillip M Young \\ Department of Radiology, Mayo Clinic, \\ Rochester, MN, USA
}

\begin{abstract}
Peripheral vascular malformations (VMs) are complex and diverse vascular lesions which require individualized pretreatment planning. Pretreatment imaging using various modalities, especially magnetic resonance imaging and time-resolved magnetic resonance angiography, is a valuable tool for classifying peripheral VMs to allow proper diagnosis, demonstrate complete extent, identify the nidus, and distinguish between low-flow and high-flow dynamics that determines the treatment approach. We discuss pretreatment imaging findings in four patients with peripheral VMs and how diagnostic imaging helped guide management.
\end{abstract}

Keywords: time-resolved MRA, cartesian acquisition with projection-like reconstruction, endovascular treatment, magnetic resonance angiography

\section{Case reports Case I}

A 3 year old girl initially presented at an outside institution with "bruising" on her left shoulder at 3 months of age. She was diagnosed with an infantile hemangioma, which did not respond to subsequent standard treatment consisting of six laser therapy treatments and 8 months of propranolol. Continued enlargement of the lesion involving her left shoulder, with worsening pain, prompted presentation to our institution for further evaluation. Magnetic resonance imaging (MRI) was performed to evaluate for an underlying vascular malformation (VM). This showed multiple small foci of T2 hyperintensity isolated to the dermis, with no underlying VM present (Figure 1). The MRI results led to a dermatology consultation and subsequent skin biopsy, with pathology showing Kaposiform hemangioendothelioma. Proper treatment ensued with oncology consultation, and vincristine chemotherapy. Follow-up consultation 3 years later noted decreased size of the left shoulder lesion with resolution of pain.

\section{Case 2}

A 26 year old male runner with long-standing anterior right knee pain for the preceding 7 years was initially evaluated in a sports medicine clinic and diagnosed with patellofemoral pain syndrome and mild lateral patellar maltracking. An MRI, performed to assess cartilaginous structures of the right knee, showed a partially intra-articular venous VM along the medial aspect of the right knee joint (Figure 2A and B) with no arterial involvement. The patient's pain reached a point where he could hardly get out of bed to put clothes on, or walk to the freezer to get ice to put on the knee. Sclerotherapy was offered and subsequently performed during three serial sessions. During the
Correspondence: Joshua B Johnson Mayo Clinic Department of Radiology, 200 First Street SW, Rochester, MN 55905, USA

$\mathrm{Tel}+\mathrm{I} 65 \mid 246400$ I

Fax +I 5072933680

Email johnson.joshual@mayo.edu 


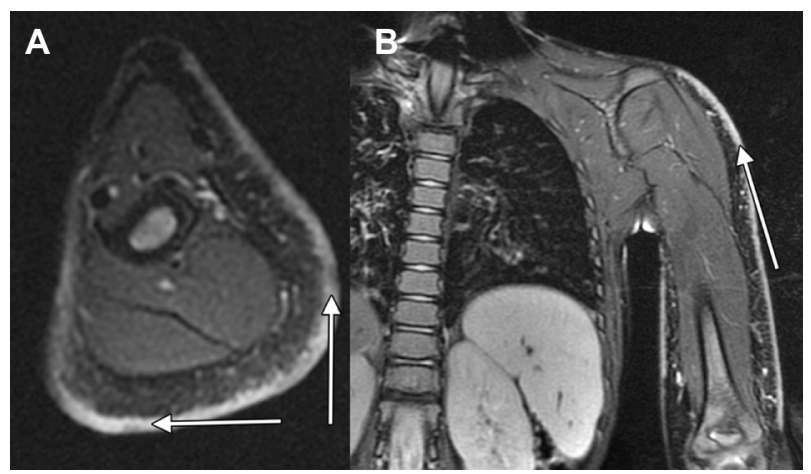

Figure I MRI images of a left arm superficial vascular lesion initially suspected as an infantile hemangioma but ultimately proved to be Kaposiform hemangioendothelioma.

Notes: Axial (A) and coronal (B) fat saturated T2 MRI scans depict multiple small foci of T2 hyperintensity within the dermis (arrows) most confluent around the mid upper arm with no extension into the subcutaneous tissues, muscles, or bones. No underlying vascular malformation is evident.

Abbreviation: MRI, magnetic resonance imaging.

first sclerotherapy (Figure 2C), $10 \mathrm{cc}$ of sodium tetradecyl sulfate 3\% (Sotradecol ${ }^{\circledR}$; AngioDynamics, Inc., Latham, NY, USA) was mixed, using foam technique, and injected into the lesion, avoiding a component involving the weight-bearing articular surface to avoid potential development of secondary osteoarthritis. Foam technique involves mixing the solution back and forth between two syringes attached to a three-way stopcock. The second sclerotherapy, 5 months later, utilized $10 \mathrm{cc}$ of Sotradecol distributed amongst three separate areas of the VM. A follow-up MRI after the second sclerotherapy depicted reduced size of the VM (Figure 2D). A third sclerotherapy was performed 4 months after the second for residual pain, using $2 \mathrm{cc}$ of Sotradecol with immediate stasis of venous flow after injection. At a 3-month follow-up appointment following the third sclerotherapy, the patient noted entering a military officer training program and being able to run $3.2 \mathrm{~km}$ (2 miles) twice weekly, pain-free.

\section{Case 3}

A 34 year old man was initially diagnosed with a left forearm VM at 14 years of age. Multiple courses of treatment including surgical resection and endovascular sclerotherapy ensued, following a typical complex recurrent and indolent course for a VM. He presented to our institution with constant pain in his left forearm, requiring scheduled narcotic pain medication. An MRI was performed, which showed a low-flow VM with a clear nidus in the interosseous membrane region of the distal forearm (Figure 3A, and B). Endovascular sclerotherapy was offered. A pre-sclerotherapy diagnostic ultrasound of the region depicted suitable percutaneous access of the VM. Subsequent nidus-targeted treatment with endovascular sclerotherapy (Figure 3C) using ultrasound for access, markedly improved symptoms after three serial treatments. During the first sclerotherapy, the venous malformation was shown to communicate with normal deep veins. Finger compression was used to occlude the deep communicating perforator veins to induce stasis in the VM, which was treated with $4 \mathrm{cc}$ of Sotradecol. During the second sclerotherapy 5 months later, the existing VM was much smaller than during the prior treatment. Again, the communicating perforator veins were manually occluded and sclerotherapy using $4 \mathrm{cc}$ of Sotradecol was performed. A third sclerotherapy a few months later utilized $4 \mathrm{cc}$ of Sotradecol. Following the sclerotherapy treatments, a follow-up MRI depicted substantially decreased size of the VM (Figure 3D). Also, the patient noted he no longer required scheduled narcotic pain medication.

\section{Case 4}

A 27-year-old woman, diagnosed at age 6 with a right hand $\mathrm{VM}$, presented to our institution for further management with increased discomfort, and concern for risk of rebleeding

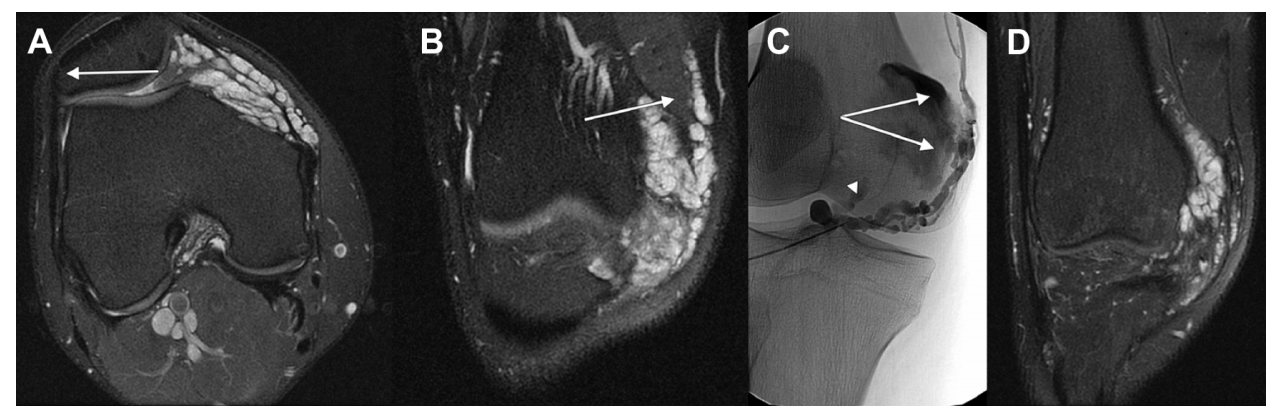

Figure $2 \mathrm{MRI}$ and fluoroscopic images of a right knee vascular malformation.

Notes: Axial (A) and coronal (B) T2 fat saturated MRI scans prior to sclerotherapy depict an undulating, partially intra-articular vascular malformation surrounding the medial patellar retinaculum with resultant lateral patellar tilt (arrow, A) and extension to the vastus medialis (arrow, B). Angiogram (C) obtained during sclerotherapy of two areas of the vascular malformation (arrows, C) with sodium tetradecyl sulfate $3 \%$ (Sotradecol ${ }^{\circledR}$; AngioDynamics, Inc., Queensbury, NY, USA). A third area (arrowhead, C) was not treated given its location involving the intra-articular weight-bearing surface. Follow-up coronal T2 MRI scan (D) following three serial sclerotherapy sessions depicts reduced size of the vascular malformation.

Abbreviation: MRI, magnetic resonance imaging. 

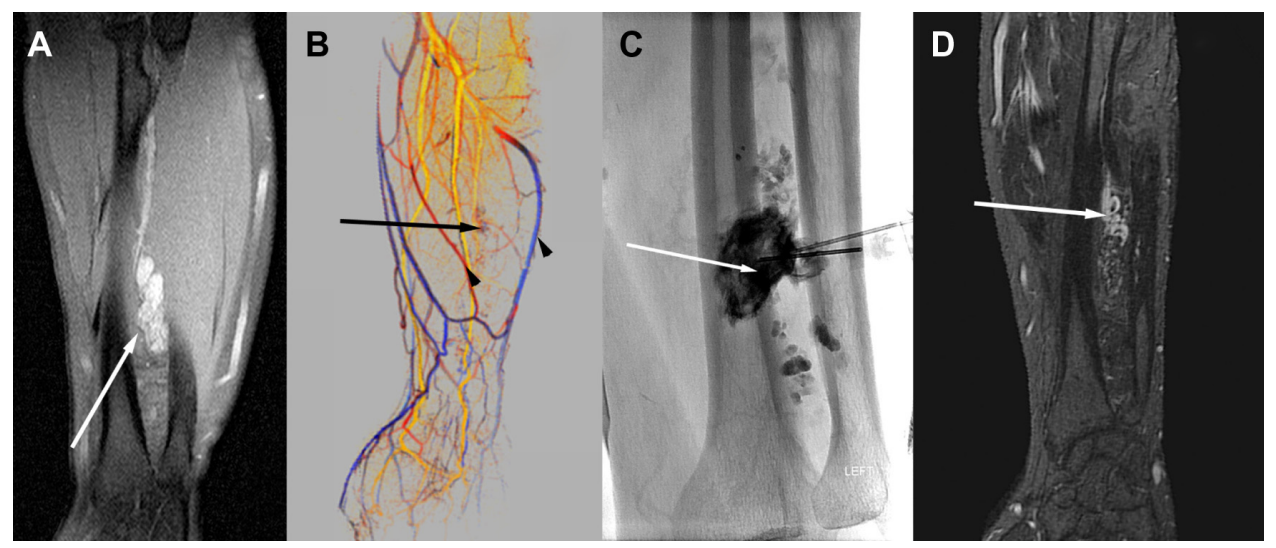

Figure 3 MRI, CAPR and fluoroscopic images of a left forearm vascular malformation.

Notes: Coronal pre-contrast TI MRI scan (A) prior to sclerotherapy depicts a low-flow vascular malformation in the distal left forearm with a nidus (arrow, A) adjacent to the interosseous membrane and patent proximal feeding vessels. A cartesian acquisition projection-like reconstruction (CAPR) time-of-arrival map (B), which depicts arterial inflow in red and yellow hues and venous outflow in blue and purple hues on a single image, again shows the nidus (arrow, B). The lack of simultaneous arterial enhancement (not shown) and normal caliber vessels (arrowheads, B) denote a low-flow lesion. Fluoroscopic image during sclerotherapy (C) with sodium tetradecyl sulfate 3\% (Sotradecol ${ }^{\circledast}$; AngioDynamics, Inc., Queensbury, NY, USA) after ultrasound-guided vascular access targeted the nidus adjacent to the interosseous membrane (arrow). Two similar sclerotherapy sessions followed. A follow-up MRI contrast-enhanced image after the third sclerotherapy (D) depicts substantially decreased size of the distal left forearm vascular malformation (arrow).

Abbreviation: MRI, magnetic resonance imaging.

given newly increased distention of her right hand veins. She had four different operations in the past to control a significant spontaneous bleeding episode, with minimal to no improvement in pain symptoms. A compression garment had been tried only intermittently in the past, with some relief. A time-resolved magnetic resonance angiography (MRA) was performed to further characterize the VM, which depicted a high-flow VM (Figure 4) with arterial supply from superficial palmar arch branches of both the ulnar and radial artery. Endovascular therapy was offered. However, given the substantial risk of collateral damage to adjacent nerves, skin, and muscle and potential loss of limb function, the patient elected for more consistent use of a compression garment prior to more invasive management.

\section{Discussion}

VMs are rare congenital anomalies. VMs are present at birth and grow as the patient does, demonstrating a normal rate of endothelial turnover. They may not become apparent until adulthood, once they enlarge enough to be visualized or produce symptoms. ${ }^{1}$ Histologically, they encompass a spectrum of lesions that include arterial, capillary, venous, and lymphatic components in various combinations, with the majority consisting of venous-predominant low-flow malformations. ${ }^{2}$
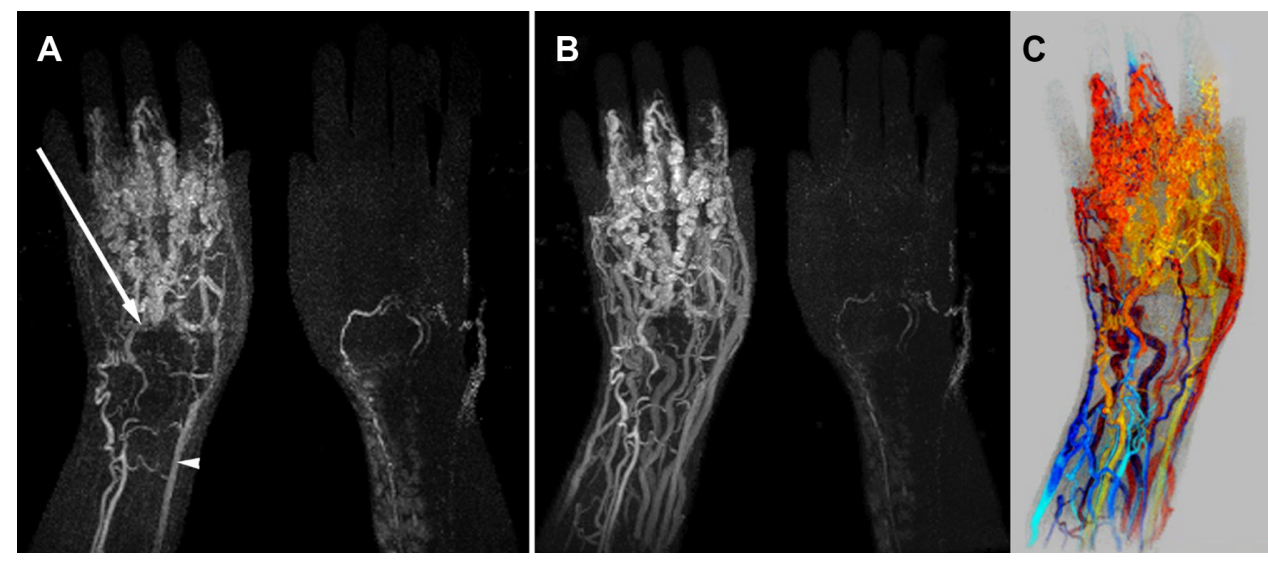

Figure 4 MRA and CAPR images of a right hand high-flow vascular malformation.

Notes: MRA images at time $0 \mathrm{sec}(\mathbf{A})$ and time $3.5 \mathrm{sec}(\mathbf{B})$ depict a right hand high-flow arteriovenous malformation with a primary arterial supply from the superficial arch (arrow, A) and a prominent radial artery (arrowhead, A) with rapid arterial flow compared to contralateral hand. Early venous drainage was through the superficial tissues of the fingers and hands. CAPR time-of-arrival map (C), which depicts arterial inflow in red and yellow hues and venous outflow in blue and purple hues on a single image, shows the dilated tortuous vessels of the high-flow arteriovenous vascular malformation.

Abbreviations: MRA, magnetic resonance angiography; CAPR, cartesian acquisition projection-like reconstruction; sec, seconds. 
Management of VMs requires a multidisciplinary approach due to their prolonged and often progressive clinical course. This typically involves multiple physicians with expertise in internal medicine, pediatric cardiology, dermatology, surgery, psychiatry, and interventional radiology, in order to correctly classify VMs and guide long-term treatment. ${ }^{3,4}$

Treatment is mainly guided by patient symptoms and classification of the VM based on low-flow versus high-flow. Additional considerations include patient age, life expectancy, comorbid conditions and progression of symptoms. Mild symptoms are typically treated more conservatively with either observation, or compression garments. Severe symptoms such as disabling pain, swelling, functional impairment, ulceration, or severe bleeding often require more invasive treatment, which is typically endovascular treatment with embolization or sclerotherapy versus surgical resection. Both treatment approaches complement each other, and are generally palliative rather than curative, requiring much dedication..$^{5}$ Low-flow VMs, which represent about $90 \%$ of VMs, can be successfully treated using endovascular sclerotherapy alone. ${ }^{4}$ This involves percutaneous access to the lesion and direct injection of a sclerosing agent, causing precipitation of endothelium proteins via denaturation and dehydration, and ultimately thrombosis and endothelial destruction. Maintaining deep vein patency is essential to provide adequate drainage of the extremity. Therefore, one must not obstruct all major venous pathways of the affected extremity with sclerotherapy. Less common high-flow VMs, defined as such by containing an arterial component, often require more aggressive treatment secondary to shunting from high-output failure states, which is associated with increased morbidity and mortality. ${ }^{6}$ High-flow VMs are typically treated first with transarterial embolization, which involves occlusion of the vascular inflow and/or outflow for primary treatment alone, or as an adjunctive treatment, prior to subsequent sclerotherapy or surgery.

Determining pertinent VM characteristics, including classification of high-flow or low-flow, is important prior to intervention. Noninvasive imaging, particularly MRI, plays an important role in the characterization of VMs. Classification into low-flow or high-flow dynamics provides a useful way to assist the interventional radiologist in planning treatment. ${ }^{7}$ Whether dealing with low-flow or high-flow VMs, it is important to identify the nidus, which represents the abnormal focus of tortuous vessels at the center of the VM. There may be multiple nidi in a single lesion. By targeting the nidus during treatment, the most direct pathways of blood flow into the malformation are sclerosed and a longer duration of effect is observed. Occasionally, an intervention can stimulate vascular endothelial cells and paradoxically worsen symptoms. Therefore targeting treatment to the nidus and thoughtfully limiting damage to adjacent structures is generally considered the best treatment approach. ${ }^{4}$ Pretreatment imaging also helps to identify the nidus for targeting treatment (Figure 3). Images can depict VM extent and associated involvement of muscle, bone, joints, or subcutaneous tissue that may predispose to complications (Figure 2). ${ }^{6}$ Though clinically apparent, the extent of a VM is often underestimated by physical exam alone. One study revealed that in greater than $75 \%$ of cases there was more extensive involvement demonstrated on MRI compared to the physical exam and nearly half of those cases resulted in a change in management. ${ }^{8} \mathrm{MRI}$ also guides therapy further by characterizing vascular inflow and outflow; determining the number of feeding vessels avoids potential problems with undertreatment of a single vessel when multiple vessels contribute.

While MRI is the mainstay of diagnostic imaging assessment of VMs, additional imaging modalities may be helpful. MRI techniques have the benefit of excellent soft tissue characterization and lack of ionizing radiation in patients who are often young and require serial follow-up. Risks of MRI include safety concerns related to the strong magnetic field, as well as the risk of nephrogenic systemic fibrosis with the use of intravenous gadolinium in patients with chronic kidney disease. ${ }^{9}$ Ultrasound provides real-time visualization during intervention for vascular access and real-time arterial and venous flow velocities. Ultrasound is limited by inability to assess deep lesions or those adjacent to bone or air. Computed tomography (CT) and CT angiography provides detail using contrast enhancement and allows assessment of calcification or thrombus, in addition to providing complementary imaging information when MR does not provide a clear answer. CT is limited by exposure to ionizing radiation, and depicts less blood flow information when compared to MR. Conventional angiography provides the concomitant treatment possibility, while it is limited by its invasive nature, projection format, and exposure to ionizing radiation. ${ }^{4}$

At our institution, we begin our VM MRI exams with fat suppressed T2-weighted sequences in the axial plane to document VM location for subsequent time-resolved contrast-enhanced images. Then, we inject a gadoliniumbased contrast material, usually the blood pool agent gadofosveset trisodium (Ablavar ${ }^{\circledR}$; Lantheus Medical Imaging, North Billerica, MA, USA) and acquire time-resolved contrastenhanced MRA images. In the extremities, we typically use specialized receiver coils and the cartesian acquisition with 
projection-like reconstruction (CAPR) technique, ${ }^{10}$ which allows creation of both time-resolved three-dimensional (3D) datasets and time-of-arrival maps ${ }^{11}$ that depict flow information on a single image (Figures $3 \mathrm{~B}$ and $4 \mathrm{C}$ ). CAPR is a $3 \mathrm{D}$ gradient echo sequence that uses elliptical centric sampling, ${ }^{12}$ two-dimensional (2D) homodyne reconstruction, ${ }^{13}$ view-sharing, ${ }^{14}$ and 2D sensitivity-encoding. ${ }^{15} \mathrm{Clinical}$ advantages of CAPR include images with both high temporal resolution that allows visualization and clear distinction of arterial and venous phases and high spatial resolution (1 $\mathrm{mm}$ isotropic) that allows sharp depiction of small branching vessels. Time-of-arrival maps allow a depiction of the $\mathrm{VM}$ on a single image, which patients generally find helpful for understanding subsequent treatment planning and management. Time-resolved MRA using CAPR technique has been shown to demonstrate flow characteristics to guide treatment. ${ }^{16,17}$ Other time-resolved techniques can be used as well, with or without view-sharing. We typically use non-view-shared, multiphase, breath-hold, 2D accelerated MRA when imaging the abdomen or pelvis and view-shared techniques, such as CAPR, in the extremities. Finally, delayed post-contrast T1-weighted fat-suppressed high-resolution gradient echo images are acquired in the blood pool phase. These are useful to document the extent of prior ablation or treatment, assess for venous thrombus or outflow obstruction, and further assess soft tissue extent. An example protocol with parameters is summarized in Table $1 .{ }^{16}$ Our studies were performed on a 3.0-T GE Scanner platform system (GE Healthcare, Waukesha, WI, USA).

The cases presented here provide different examples of how pretreatment imaging guided subsequent management. This included MRI depicting lack of an actual underlying VM leading to skin biopsy, and the correct diagnosis and treatment (Figure 1); MRI depicting the full extent of the underlying VM in relation to sensitive structures, therefore limiting sclerotherapy to safe regions and avoiding possible secondary osteoarthritis (Figure 2); MRI depicting a clear single nidus of the VM, which was targeted for subsequent sclerotherapy (Figure 3); and MRI confirming the high-flow nature of a VM (Figure 4), with endovascular embolization and sclerotherapy offered, but ultimately managed conservatively with compression garment given the clinical setting. These are only a subset of the advantages pretreatment imaging can provide for these complex vascular lesions. Additional benefits have been described previously, and include determining the focal versus diffuse nature of a VM which may therefore require multiple treatment sessions; fully characterizing both the superficial and deep venous system drainage of an extremity to ensure adequate residual drainage following treatment, particularly in patients with Klippel-Trénaunay syndrome with abnormal underlying venous systems; and defining the VM connection with the deep venous system to determine the risk of deep venous thrombosis following sclerotherapy. ${ }^{6}$

\section{Conclusion}

Peripheral VMs are complex vascular lesions that consist of a variety of combinations of arterial, capillary, venous, and/or lymphatic vessels. Pretreatment imaging, primarily with MRI, provides valuable information prior to intervention in order to make the proper diagnosis, determine lesion extent, identify the nidus, and classify flow dynamics into low-flow or highflow to guide subsequent treatment. In particular, imaging with MRI and time-resolved MRA provides both high spatial and temporal resolution allowing for precise lesion characterization and treatment planning. This may allow for more effective treatment of VMs and thus improved patient outcomes.

Table I Example MRI protocol for CAPR MRA of the forefoot for 3.0-T systems

\begin{tabular}{|c|c|c|c|}
\hline Parameter & Axial T2 with fat suppression & Time-resolved contrast-enhance MRA (CAPR) & Post-contrast TI LAVA \\
\hline Acquisition format & 2D multi-slice & $3 \mathrm{D}$ & $3 \mathrm{D}$ \\
\hline $\mathrm{TR}$ (msec) & $3,000-5,000$ & $5-6$ & $6-7$ \\
\hline $\mathrm{TE}(\mathrm{msec})$ & $45-80$ & $2-3$ & $1-2$ \\
\hline Flip angle (degrees) & 90 & 30 & 12 \\
\hline Thickness (mm) & 4 & 1 & 3 \\
\hline NEX & 2 & 1 & 1 \\
\hline Matrix & $320 \times 256$ & $400 \times 400$ & $384 \times 224$ \\
\hline FOV & 260 & 300 & 350 \\
\hline $\begin{array}{l}\text { 2D SENSE acceleration } \\
\text { factor }\end{array}$ & 1 & 8 & 1 \\
\hline Acquisition time (sec) & $5-6 \min$ & $6 \mathrm{sec}$ per 3D time frame continued for $2.5 \mathrm{~min}$ & $30 \mathrm{sec}$ \\
\hline
\end{tabular}

Note: 3.0-T (GE Healthcare, Waukesha, WI, USA).

Abbreviations: 2D, two-dimensional; 3D, three-dimensional; CAPR, cartesian acquisition projection-like reconstruction; FOV, field of view; LAVA, liver acquisition with volume acceleration; min, minutes; MRA, magnetic resonance angiography; NEX, number of excitations; sec, seconds; SENSE, sensitivity encoding; TE, echo time; TR, repetition time; MRI, magnetic resonance imaging. 


\section{Acknowledgment}

Support from National Institute of Health (NIH) Grants EB000212, HL070620, and RR018898 was used for this work.

\section{Disclosure}

Mayo Clinic has received licensing revenue for technologies used in this work from GE Healthcare (Waukesha, WI, USA) and Toshiba (Tustin, CA, USA). Stephen J Riederer, $\mathrm{PhD}$, is currently receiving federal NIH grants (\#EB000212, HL070620). The other authors report no conflicts of interest in this work.

\section{References}

1. Mulliken JB, Glowacki J. Hemangiomas and vascular malformations in infants and children: a classification based on endothelial characteristics. Plast Reconstr Surg. 1982;69(3):412-422.

2. Enjolras O, Wassef M, Chapot R. Introduction: ISSVA classification. In: Enjolras O, Wassef M, Chapot R, editors. Color Atlas of Vascular Tumors and Vascular Malformations. New York: Cambridge University Press; 2007:1-11.

3. Donnelly LF, Adams DM, Bisset GS 3rd. Vascular malformations and hemangiomas: a practical approach in a multidisciplinary clinic. AJR Am J Roentgenol. 2000;174(3):597-608.

4. Hyodoh H, Hori M, Akiba H, Tamakawa M, Hyodoh K, Hareyama M. Peripheral vascular malformations: imaging, treatment approaches, and therapeutic issues. Radiographics. 2005;25(1):S159-S171.

5. Lee BB, Laredo J, Kim YM, Neville R. Congenital vascular malformations: general treatment principles. Phlebology. 2007;22(6):258-263.

6. Fayad LM, Hazirolan T, Bluemke D, Mitchell S. Vascular malformations in the extremities: emphasis on MR imaging features that guide treatment options. Skeletal Radiol. 2006;35(3):127-137.
7. Moukaddam H, Pollak J, Haims AH. MRI characteristics and classification of peripheral vascular malformations and tumors. Skeletal Radiol. 2009;38(6):535-547.

8. Rinker B, Karp NS, Margiotta M, Blei F, Rosen R, Rofsky NM. The role of magnetic resonance imaging in the management of vascular malformations of the trunk and extremities. Plast Reconstr Surg. 2003;112(2):504-510.

9. Daftari Besheli L, Aran S, Shaqdan K, Kay J, Abujudeh H. Current status of nephrogenic systemic fibrosis. Clin Radiol. 2014;69(7): 661-668.

10. Haider CR, Hu HH, Campeau NG, Huston J 3rd, Riederer SJ. 3D high temporal and spatial resolution contrast-enhanced MR angiography of the whole brain. Magn Reson Med. 2008;60(3):749-760.

11. Riederer SJ, Haider CR, Borisch EA. Time-of-arrival mapping at three-dimensional time-resolved contrast-enhanced MR angiography. Radiology. 2009;253(2):532-542.

12. Wilman AH, Riederer SJ. Performance of an elliptical centric view order for signal enhancement and motion artifact suppression in breath-hold three-dimensional gradient echo imaging. Magn Reson Med. 1997;38(5):793-802.

13. Noll DC, Meyer CH, Pauly JM, Nishimura DG, Macovski A. A homogeneity correction method for magnetic resonance imaging with timevarying gradients. IEEE Trans Med Imaging. 1991;10(4):629-637.

14. Riederer SJ, Tasciyan T, Farzaneh F, Lee JN, Wright RC, Herfkens RJ. MR fluoroscopy: technical feasibility. Magn Reson Med. 1988;8(1):1-15.

15. Weiger M, Pruessmann KP, Boesiger P. 2D SENSE for faster 3D MRI. MAGMA. 2002;14(1):10-19.

16. Mostardi PM, Young PM, McKusick MA, Riederer SJ. High temporal and spatial resolution imaging of peripheral vascular malformations. J Magn Reson Imaging. 2012;36(4):933-942.

17. Haider CR, Glockner JF, Stanson AW, Riederer SJ. Peripheral vasculature: high-temporal- and high-spatial-resolution threedimensional contrast-enhanced MR angiography. Radiology. 2009; 253(3):831-843.
Journal of Vascular Diagnostics

\section{Publish your work in this journal}

Journal of Vascular Diagnostics is an international, peer-reviewed journal of diagnostics, focusing on non invasive vascular investigation methods involved in the evaluation of vascular diseases. The journal is committed to the rapid publication in the fields of vascular diseases. Original research, review, case reports, expert opinion and commentaries

\section{Dovepress}

are all considered for publication. The manuscript management system is completely online and includes a very quick and fair peer-review system, which is all easy to use. Visit http://www.dovepress.com/testimonials.php to read real quotes from published authors. 